\title{
Sweet's syndrome associated with myelogenous leukemia and pulmonary involvement
}

\section{Tho the Editors:}

Sirs,

Sweet's syndrome is characterised by acute onset of fever, leukocytosis and erythematous plaque infiltrated by neutrophils. It can be associated with infections, autoimmune diseases, inflammatory bowel disease, malignancy and drugs, but the pathogenesis is still unknown. Approximately $20-25 \%$ of patients with Sweet's syndrome have an associated malignancy [1], mostly hematopoietic (myelodysplastic syndromes and acute myeloid leukaemia) [2]. Pulmonary involvement in Sweet's syndrome is uncommon, having been reported in only few cases in literature $[3,4]$ and it seems to be more frequently associated with hematologic dyscrasia [5].

We report a case of Sweet's syndrome associated with myelogenous leukaemia and respiratory involvement. The patient had died at the time of writing. A 83-year-old non smoker woman was admitted to hospital due to persistent fever, cough and dyspnea; she had a two-month history of generalised fatigue and progressive breathlessness on exertion. Blood tests were remarkable for Neutrophil Leukocytosis (White Blood Cells 66.1 $\times 10^{\wedge} 9 / \mathrm{L}$, Neutrophils, 30.8 $\times 10^{\wedge} 9 / \mathrm{L}$ ) and increased C-reactive protein and Erythrocyte sedimentation (159.1 $\mathrm{mg} / \mathrm{L}$ and $120 \mathrm{~mm} / \mathrm{h}$ respectively).

A chest xray showed bilateral pleural effusion predominant on the right side and bilateral pulmonary infiltrates. The CT scan confirmed the presence of bilateral parenchimal infiltrates with bilateral pleural effusion, more predominant on the

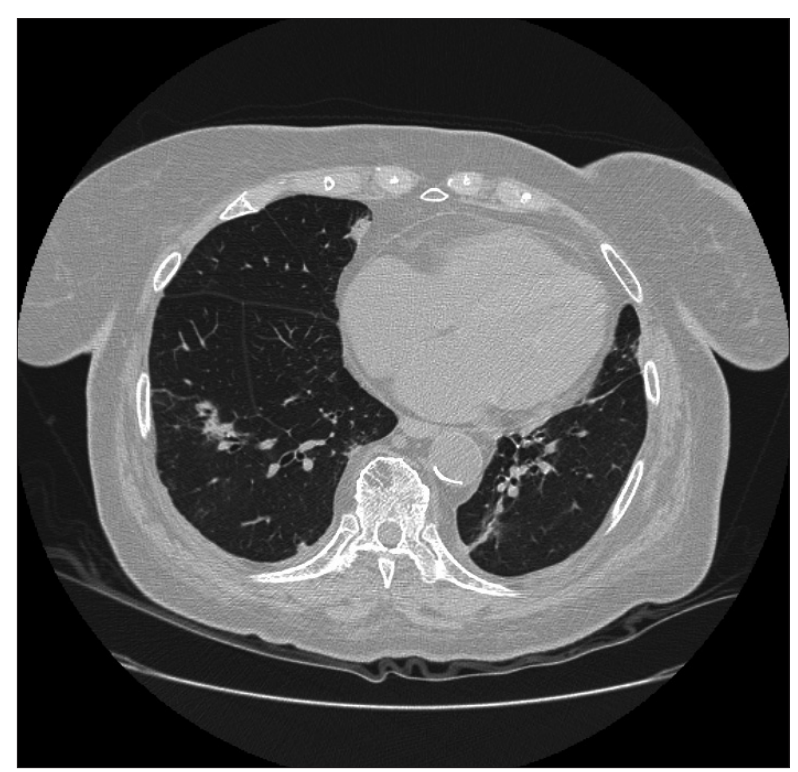

Fig. 1. - Chest CT scan showing parenchimal infiltrates with not significant lymphoadenopathy. right, and left pleural thickening $(5 \times 1.5 \mathrm{~cm})$ with no significant lymphadenopathy (figure 1).

A fibre-optic bronchoscopy showed no endobronchial abnormalities; results of gram, fungal and acid-fast bacilli staining and search for viruses in bronchial washing were negative. Investigations for infections (blood, urine and bronchial samples, serology) were negative. The patient underwent various successive antibiotic treatments but her condition progressively worsened requiring high oxygen-therapy. A fever persisted with an intermittent pattern and we noted a new painless cutaneous plaque, with a $5 \mathrm{~cm}$ diameter, in the right upper thoracic quadrant; which appeared red/violet and roundish, and progressively increased in size, developing also postulation and ulceration (figure 2). A skin biopsy showed a dense neutrophilic infiltrate within the superficial dermis without necrotising vasculitis and a diagnosis of Sweet's syndrome was made.

Bone marrow biopsy and aspirate were performed; an acute myelomonocytic leukemia was diagnosed and cytogenetic analysis revealed translocation $(6 ; 9)(\mathrm{p} 23 ; \mathrm{q} 34)$, which is mainly found in specific subtypes of acute myeloid leukemia and myelodysplastic syndrome, and it is also associated with an unfavourable prognosis. Fluorescent in-situ hybridisation (FISH) analysis of interphase cells using the Vysis dual fusion $\mathrm{BCR}$ and $\mathrm{ABL}$ gene probes did not detect gene fusion.

The patient was placed on Gleevec therapy and steroids but unfortunately her condition deteriorated rapidly and she passed away a few weeks later. The autopsy revealed multiple parenchimal lung infiltrates with intra-alveolar infiltrates of neutrophils (possible pulmonary localization of Sweet's

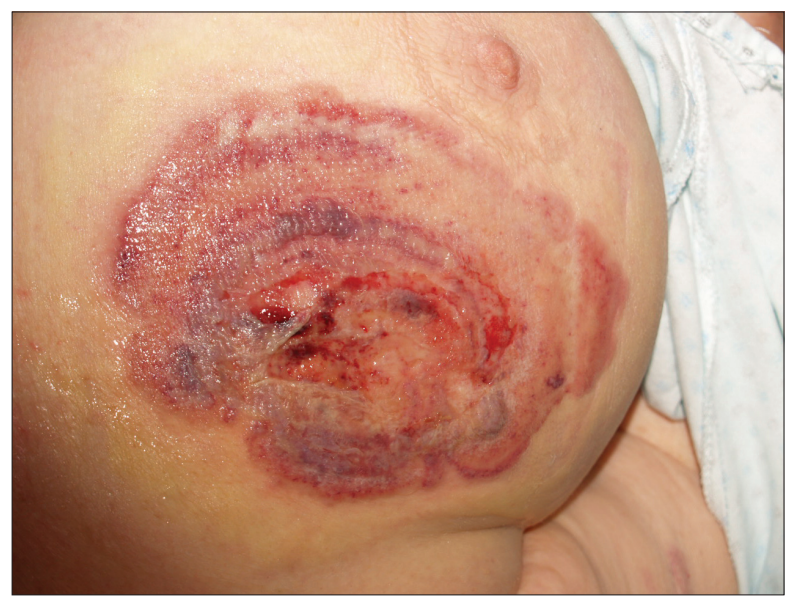

Fig. 2. - Red/violet cutaneous plaque (diameter of $5 \mathrm{~cm}$ ) in the right upper thoracic quadrant, with postulation and ulceration. 


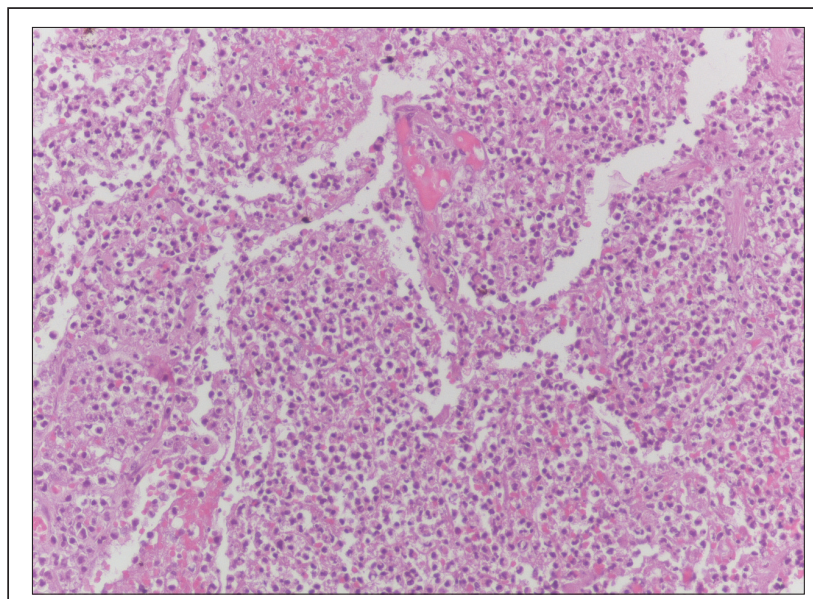

Fig. 3. - Autopsy specimen of the lung (Hematoxylin Eosin 20X) showing multiple parenchimal lung infiltrates with intra-alveolar infiltrates of neutrophils.

syndrome) (figure 3), a subverted anatomical structure of bone marrow and spleen parenchyma for the presence of a cell population consisting of elements of myeloid medium size type compatible with myeloid myelomonocytic leukaemia and splenic localisation of granulocytic leukaemia (chloroma).

The characteristic eruption of Sweet's syndrome can occur before, concomitantly, or after pulmonary symptoms. Thoracic imaging can show unilateral or bilateral interstitial infiltrates, nodules, cavitary lesions or pleural effusions [5]. Parenchymal lung histology usually reveal intraalveolar infiltrates of neutrophils [6]; less commonly it can be associated with organising pneumonia [7].

Although rare, Sweet's syndrome can be very important in the differential diagnosis of fever of unknown origin with neutrophilia. The most frequent occult malignancies to cause fever are of reticulo-endothelial origin (eg. lymphoma and leukaemia) and this case highlights the importance of making an accurate and timely diagnosis of Sweet's syndrome mostly in the elderly as it is frequently associated with malignancies.

\section{Claudia Ravaglia1, Giovanni Poletti², Carlo Gurioli1, Gian Luca Casoni1, Alessandra Dubini ${ }^{3}$, Giuseppe $\operatorname{Re}^{4}$, Venerino Poletti ${ }^{1}$}

\author{
1 Department of Diseases of the Thorax, G.B. Morgagni - \\ L. Pierantoni Hospital, Forli, \\ 2 Department of Clinical Pathology Area Vasta Romagna, \\ 3 Pathology Service, G.B. Morgagni - L. Pierantoni \\ Hospital, Forli, \\ 4 Department of Internal Medicine, Umberto I Hospital, \\ Lugo, Italy.
}

Correspondence: Dr. Claudia Ravaglia, Interventional Pulmonology, Department of Thoracic Diseases, G.B. Pierantoni - L. Morgagni Hospital, Forli, Italy; e-mail: claudiaravaglia@alice.it

\section{References}

1. Cohen PR, Talpaz M, Kurzrock R. Malignancy-associated Sweet's syndrome: review of the world literature. J Clin Oncol 1988; 6: 1887-1897.

2. Cohen PR, Holder WR, Tucker SB, Kono S, Kurzrock R. Sweet's Syndrome patients with solid tumors. Cancer 1993; 72: 2723-2731.

3. Robbins CM, Mason SE, Hughey LC. Sweet syndrome with pulmonary involvement in a healthy young woman. Arch Dermatol 2009; 145: 344-346.

4. Xu HH, Xiao T, Gao XH, Chen HD. Ulcerative Sweet syndrome accompanied by interstitial lung disease and myelodysplastic syndrome. Eur J Dermatol 2009; 19: 411-412.

5. Astudillo L, Sailler L, Launay F, et al. Pulmonary involvement in Sweet's syndrome: a case report and review of the literature. Int J Dermatol 2006; 45: 677680.

6. Bourke SJ, Quinn AG, Farr PM, Ashcroft T, Gibson GJ. Neutrophilic alveolitis in Sweet's syndrome. Thorax 1992; 47: 572-573.

7. Garg R, Soud Y, Lal R, Mehta N, Kone BC. Myelodysplastic syndrome manifesting as Sweet's Syndrome and bronchiolitis obliterative organizing pneumonia. Am J Med 2006: 119: e5-e7.

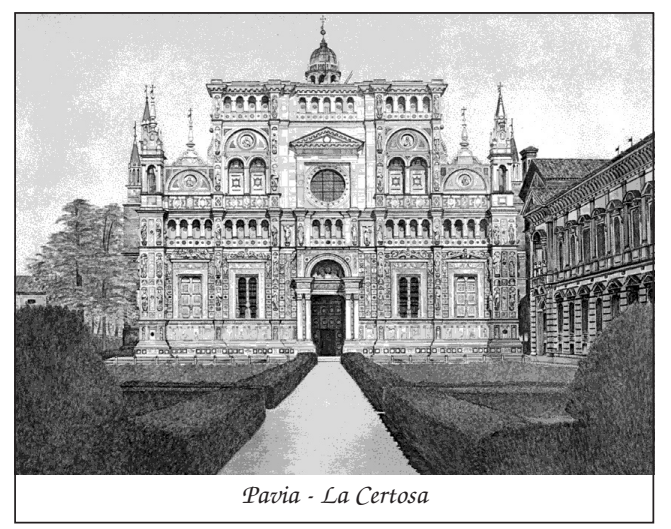

